\title{
Prospective international multicenter study on endoscopic ultrasound-guided biliary drainage for patients with malignant distal biliary obstruction after failed endoscopic retrograde cholangio- pancreatography
}

Authors

Institutions
Mouen A. Khashab ${ }^{1}$, Schalk Van der Merwe², Rastislav Kunda ${ }^{3}$, Mohamad H. El Zein ${ }^{1}$, Anthony Y. Teoh ${ }^{4}$, Fernando P. Marson ${ }^{5}$, Carlo Fabbri ${ }^{6}$, Ilaria Tarantino ${ }^{7}$, Shyam Varadarajulu ${ }^{8}$, Rani J. Modayil ${ }^{9}$, Stavros N. Stavropoulos ${ }^{9}$, Irene Peñas ${ }^{10}$, Saowanee Ngamruengphong ${ }^{1}$, Vivek Kumbhari ${ }^{1}$, Joseph Romagnuolo ${ }^{11}$, Raj Shah ${ }^{12}$, Anthony N. Kalloo', Manuel Perez-Miranda ${ }^{10}$, Everson L. Artifon ${ }^{5}$

Institutions are listed at the end of article. submitted 4. August 2015 accepted after revision 22. January 2016

\section{Bibliography}

Dol http://dx.doi.org/ 10.1055/s-0042-102648 Published online: 30.3.2016 Endoscopy International Open 2016; 04: E487-E496

(c) Georg Thieme Verlag KG Stuttgart · New York E-ISSN 2196-9736

\section{Corresponding author}

\section{Mouen A. Khashab, MD} Johns Hopkins Hospital Gastroenterology 1800 Orleans Street Suite 7125B Baltimore

MD 21287

USA

Fax: +1-443-683-8335 mkhasha1@jhmi.edu
Background and aims: Endoscopic ultrasoundguided biliary drainage (EUS-BD) has emerged as an alternative to traditional radiologic and surgical drainage procedures after failed endoscopic retrograde cholangiopancreatography (ERCP). However, prospective multicenter data are lacking. The aims of this study were to prospectively assess the short- and long-term efficacy and safety of EUS-BD in patients with malignant distal biliary obstruction.

Patients and methods: Consecutive patients at 12 tertiary centers (5 US, 5 European, 1 Asian, 1 South American) with malignant distal biliary obstruction and failed ERCP underwent EUS-BD. Technical success was defined as successful stent placement in the desired position. Clinical success was defined as a reduction in bilirubin by $50 \%$ at 2 weeks or to below $3 \mathrm{mg} / \mathrm{dL}$ at 4 weeks. Adverse events were prospectively tracked and graded according to the American Society for Gastrointestinal Endoscopy (ASGE) lexicon's severity grading system. Overall survival and duration of stent patency were calculated using Kaplan-Meier analysis.

\section{Background \\ $\nabla$}

The use of endoscopic ultrasound (EUS) in the treatment and palliation of gastrointestinal and pancreaticobiliary diseases, including EUS-guided biliary drainage (EUS-BD), is increasing at expert centers [1]. In patients with normal, non-obstructed upper gastrointestinal anatomy, selective bile duct cannulation by experts at endoscopic retrograde cholangiopancreatography (ERCP) is successful in over $90 \%$ of cases. When bile duct access is not possible due to failed cannulation, altered upper gastrointestinal tract anatomy, distorted ampulla, gastric outlet obstruction (GOO), periampullary diverticulum, or in situ enteral stents, EUS-BD has been increasingly used as a minimally invasive alternative to surgical or radiological
Results: A total of 96 patients (mean age 66 years, female $45 \%$, pancreatic cancer $55 \%$ ) underwent EUS-BD. Stent placement (technical success) was achieved in 92 (95.8\%) patients (metallic stent 84 , plastic stent 8 ). Mean procedure time was 40 minutes. Clinical success was achieved in 86 (89.5\%) patients. A total of 10 (10.5\%) adverse events occurred: pneumoperitoneum $(n=2)$, sheared wire $(n=1)$, bleeding $(n=$ $1)$, bile leak $(n=3)$, cholangitis $(n=2)$, and unintentional perforation $(n=1) ; 4$ graded as mild, 4 moderate, 1 severe, and 1 fatal (due to perforation). A total of 38 (44\%) patients died of disease progression during the study period. The median patient survival was 167 days (95\%CI $112-221)$ days. The 6-month stent patency rate was 95\% (95\%CI 94.94-95.06\%) and the 1-year stent patency was $86 \%$ (95\%CI $85.74-86.26 \%$ ).

Conclusion: This study on EUS-BD demonstrates excellent efficacy and safety of EUS-BD when performed by experts.

\section{Study registration: NCT01889953}

drainage [1]. Recent evidence suggests that EUSBD was equally effective as percutaneous transhepatic biliary drainage (PTBD) in patients with distal malignant biliary obstruction and failed ERCP and was also associated with significant cost savings [2].

Wiersema et al. first described EUS-guided biliary access in 1996 and reported seven patients who successfully underwent EUS-guided cholangiography after failed ERCP [3]. In 2001, Giovannini et al. [4] published the first case of successful EUSguided fistula creation between the duodenal bulb and the distal common bile duct (CBD) using a plastic stent in a patient with unresectable malignant biliary obstruction. Multiple studies have been published since then, reporting on the techniques and outcomes of EUS-BD [5-16]. 

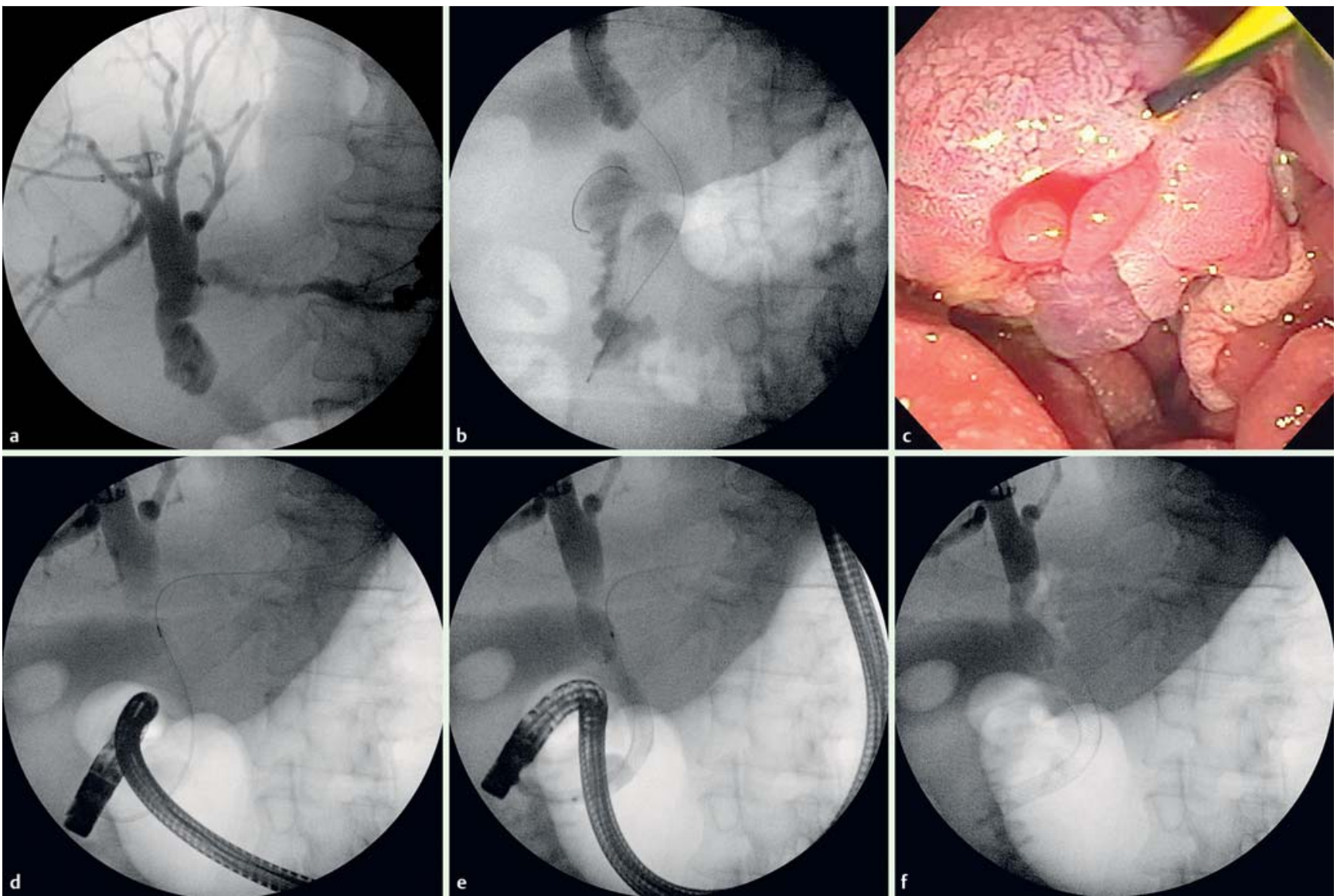

Fig. 1 Endoscopic ultrasound-guided biliary drainage (EUS-BD) using the rendezvous technique in a patient with ampullary carcinoma and two prior failed endoscopic retrograde cholangiopancreatography (ERCP) procedures. a Fluoroscopic image demonstrating a cholangiogram after puncture of the left main hepatic duct. b Successful passage of guidewire through the papilla into the duodenum. $\mathbf{c}$ Endoscopic image after the wire has been collected from the duodenum. $\mathbf{d}$ Retrograde cannulation of the common bile duct (CBD). e Fluoroscopic image during self-expandable metallic stent (SEMS) deployment. f Fluoroscopic image demonstrating transpapillary SEMS deployment.

EUS-BD can be performed by one of three methods. First, a rendezvous technique may be considered whereby a wire is placed into an intrahepatic or extrahepatic bile duct, passed through the papilla and is retrieved by a duodenoscope for biliary interventions. Second, direct transluminal stenting using a transgastric or transduodenal approach may be performed without accessing the papilla $[17,18]$. A third approach that has not been extensively reported is EUS-guided antegrade transpapillary (or trans-anastomotic) biliary stent (AGS) placement [19-21].

The major shortcoming of the current literature on safety and efficacy of EUS-BD is the absence of large prospective trials. The primary outcome of this multicenter, international prospective study (NCT01889953) was to assess the rate of clinical success of EUS-BD. Secondary outcomes included rate of technical success, rate and severity of adverse events, and long-term outcomes of EUS-BD.

\section{Patients and methods}

$\nabla$

This was an international, multicenter, prospective study at 12 tertiary centers (5 US, 5 European, 1 Asian, and 1 South American) conducted between October 2012 and November 2014. The study was approved by the Institutional Review Boards for Human Research and complied with Health Insurance Portability and Accountability Act (HIPAA) regulations at each institution. Written informed consent was obtained before study participa- tion using the IRB-approved consent form. Patients with malignant biliary obstruction and failed ERCP at all participating centers were initially referred for EUS-BD. Consecutive adult patients (18-80 years of age) with jaundice due to inoperable (by EUS and/or CT criteria or due to health status) malignant distal (greater than $2 \mathrm{~cm}$ distal to hilum) biliary obstruction and who had prior failed ERCP attempts were eligible to be enrolled in the study. Failure of ERCP was considered to be two unsuccessful attempts, according to each institution's definition of "failed" procedure. One failure at an outside institution and one failure at a participating institution were considered to be total of two failures. Some patients may have had a failed ERCP and EUS-BD under a single session.

Exclusion criteria were as follows: inability to give informed consent, life expectancy of less than 1 month, pregnant or breastfeeding women, acute gastrointestinal bleeding, uncorrectable coagulopathy defined by prothrombin time $<50 \%$ of control; partial thromboplastin time $(\mathrm{PTT})>50$ seconds, International Normalized Ratio (INR) $>1.5$, and/or platelet count $<50000$, inability to tolerate sedated upper endoscopy due to cardiopulmonary instability or other contraindication to endoscopy, prior total gastrectomy, Roux-en-Y gastric bypass, esophagectomy and sleeve gastrectomy, cirrhosis with portal hypertension, varices, and/or ascites, and liver metastases burden of more than $30 \%$. 

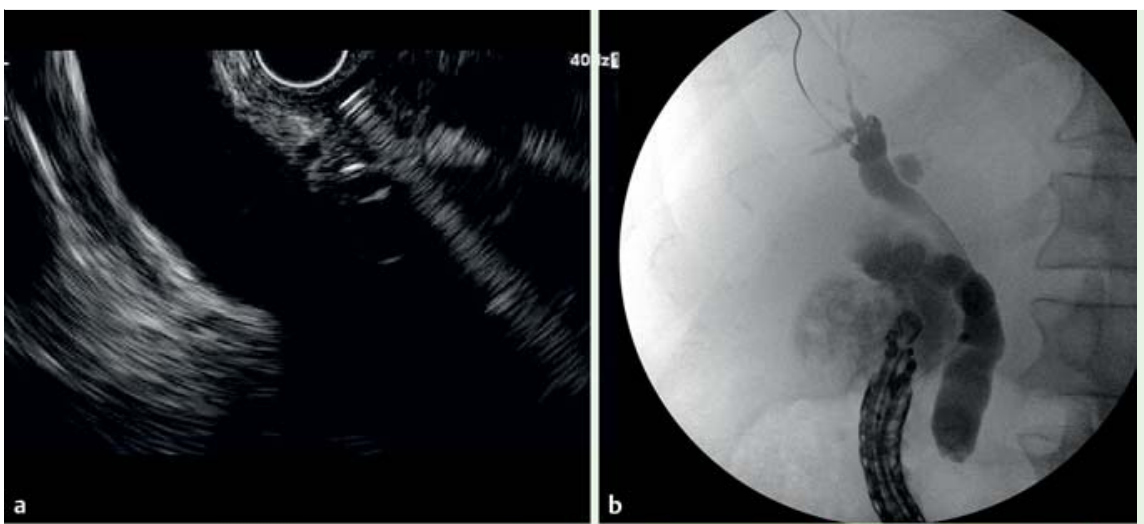

Fig. 2 Endoscopic ultrasound-guided biliary drainage (EUS-BD) with choledochoduodenostomy (CDS) in a patient with distal common bile duct (CBD) obstruction due to a cancer in the head of the pancreas. a Sonographic image demonstrating a dilated CBD about to be punctured. b Fluoroscopic image demonstrating cholangiogram and guidewire in the right hepatic duct. c Fluoroscopic image during self-expandable metallic stent (SEMS) deployment. $\mathbf{d}$ Endoscopic image of SEMS in the duodenal bulb.
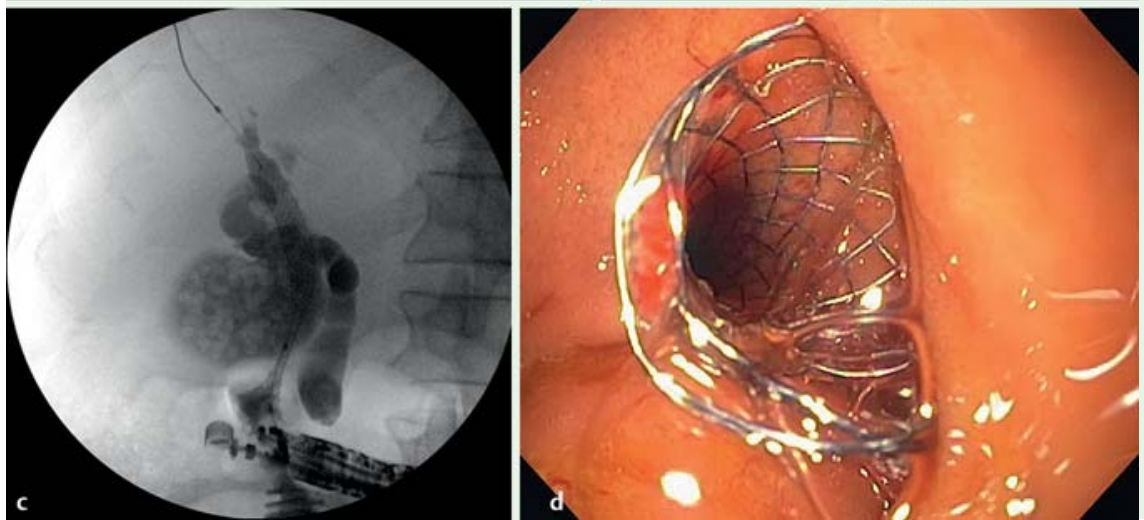

\section{Procedural algorithm and techniques}

Procedures were performed by interventional endoscopists with expertise in EUS, ERCP, and interventional EUS. All participating endoscopists had completed at least 20 prior successful EUS-BD procedures. In patients with an accessible ampulla, an initial attempt using the rendezvous technique was considered at the discretion of the endoscopist. However, if advancing the guidewire into the duodenum across the ampulla was not possible with aggressive wire manipulation, tract dilation to advance a catheter was avoided and the procedure was converted to a transluminal approach. Self-expandable metallic stents (SEMS) were preferentially placed. All procedures were performed with monitored anesthesia care, general anesthesia or endoscopist-administered propofol sedation depending on institutional sedation practices in a hospital setting with fluoroscopic capabilities. Prophylactic antibiotics, a fluoroquinolone or second generation cephalosporin, were administered before all procedures.

\section{Rendezvous technique}

A linear echoendoscope (UC140P-AL5; Olympus America, Center Valley, PA, USA; or Pentax) was used to achieve initial biliary access within a segment of dilated bile duct proximal to the site of obstruction. The tip of the echoendoscope was positioned in the gastric fundus or duodenal bulb when accessing the intrahepatic and extrahepatic bile duct, respectively. A 19-gauge or 22-gauge fine needle aspiration (FNA) needle (Echotip, Cook Medical, Winston-Salem, NC, USA; or Expect 19 Flex, Boston Scientific, Natick, MA, USA) was used to puncture the bile duct with access confirmed by aspiration of bile and cholangiogram. Depending on the needle chosen, either a 0.035-inch (Jagwire, Boston Scientific, or Glidewire, Boston Scientific), 0.025-inch (VisiGlide, Olympus), or 0.018 inch (Roadrunner, Cook Medical) guidewire was then advanced into the bile duct. The smaller 0.018-inch wires were exchanged for larger wires before stent placement. The echoendoscope and needle were angled to facilitate antegrade guidewire passage through the site of obstruction and across the papilla with coiling of the wire in the duodenum. The echoendoscope was then withdrawn leaving the guidewire in place. A side-viewing endoscope was passed to the papilla and a snare or biopsy forceps was used to grasp the guidewire and withdraw it through the endoscope with subsequent stent placement as per standard ERCP techniques ( $\bullet$ Fig. $\mathbf{1}$ ).

\section{Direct transluminal technique (choledocho-}

duodenostomy [CDS], hepatogastrostomy [HGS], hepatoduodenostomy [HDS])

In transluminal cases, the entire procedure was performed using the echoendoscope. After the bile duct was accessed as described above, the biliary-enteric fistula was dilated with a catheter or balloon, and a variety of devices were used to facilitate stent placement. These devices were selected based on the patient's anatomy and features of the obstructing stricture. Stent insertion was then performed $(\bullet$ Fig. $2, \bullet$ Fig. 3). For patients who underwent HGS, at least $3 \mathrm{~cm}$ of stent was left in the gastric lumen to avoid intraperitoneal stent migration.

\section{Antegrade stenting}

The dilated biliary ductal segment was punctured with an FNA needle and contrast was then injected through the needle to provide a cholangiogram. A hydrophilic guidewire was advanced through the needle and manipulated across the stricture. The FNA needle was then removed, and the tract was created using a 6 Fr cystotome (Cysto-Gastro set, Endo-flex, Voerde, Düsseldorf, Germany) or dilated as described above. To facilitate antegrade stent placement, the malignant stricture was dilated before advancing the stent through the therapeutic channel of the echoen- 

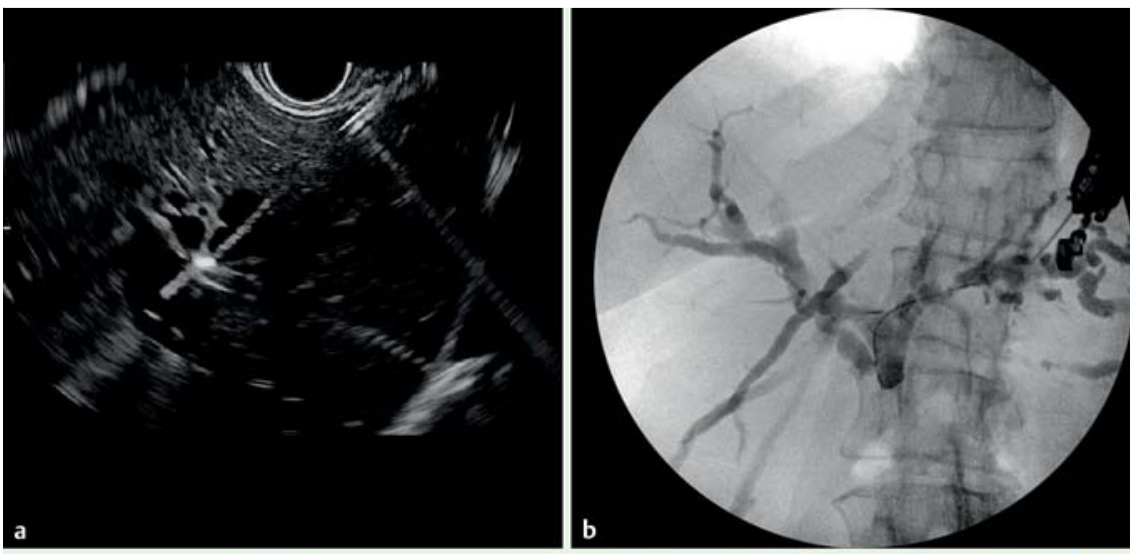

Fig. 3 Endoscopic ultrasound-guided biliary drainage (EUS-BD) with hepatogastrostomy (HGS) in a patient with Bismuth II cholangiocarcinoma. a Sonographic image of puncture of the left main hepatic duct. b Fluoroscopic image of cholangiogram and guidewire insertion. c Fluoroscopic image of self-expandable metallic stent (SEMS) deployment. d Endoscopic image of SEMS deployment. Note the proximity to the gastroesophageal junction.
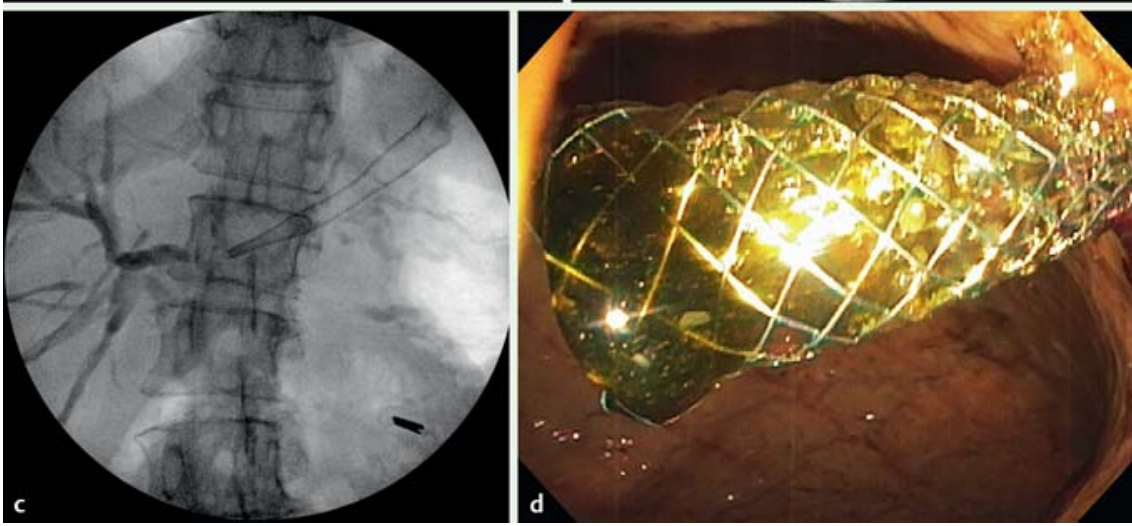

doscope over the guidewire. The stent was then deployed either across the stricture transpapillary or transanastomotic in patients with biliary-enteric anastomoses ( Fig.4) [21,22].

\section{Post-procedure care and follow-up}

All patients recovered from their procedures according to standard practice. Stable outpatients without complaints were discharged home after 3 hours of observation. Outpatients who were unstable or who were symptomatic (abdominal pain, vomiting, fever, etc.) were admitted to the hospital for observation. All patients were given a 5-day course of antibiotics. To determine the safety of EUS-BD, all pre-, intra- and immediate postprocedure adverse events were recorded. Inpatients were evaluated on a daily basis. Outpatients received a phone call on day 1 , day 7 , and day 30 from the study procedure to check for any post-procedure complications. Liver tests were checked before the procedure and then re-checked at 2 weeks and 4 weeks after the procedure.

\section{Outcomes measured and definitions}

The primary outcome of the study was rate of clinical success of EUS-BD. Clinical success was defined as a reduction in bilirubin by $50 \%$ at 2 weeks and to below $3 \mathrm{mg} / \mathrm{dL}$ (a level that allows patients to undergo chemotherapy) at 4 weeks after the procedure. Secondary outcomes were rate of technical success, safety (rate and severity of adverse events), and long-term outcomes (stent occlusion, stent migration, and survival). Technical success was defined as successful stent placement in the desired location as determined endoscopically and/or radiographically. Procedurerelated complications were recorded and included: peritonitis, symptomatic bile leak, cholangitis, bleeding, pancreatitis, intraperitoneal stent migration, subcapsular liver hematoma, pneumoperitoneum, unintentional perforation, retained sheared wire, and procedure-related death. All hospitalizations, procedures, and/or surgeries needed to treat procedure-related complications were prospectively tracked and recorded. Adverse events were graded according to the ASGE lexicon's severity grading system [23]. Procedural time was calculated from initial bile duct puncture to stent placement. Stent patency was defined as the time period between stent placement and its obstruction. Coaxial electrocautery was defined as the usage of a 6 Fr cystotome for dilation while non-coaxial electrocautery was defined as usage of a needle-knife for dilation. Type of duodenal stenosis was defined as follows: type I stenosis occurred at the level of the duodenal bulb or upper duodenal genu but without involvement of the papilla, type II stenosis affected the second part of the duodenum with involvement of the major papilla, and type III stenosis involved the third part of the duodenum distal to and without involvement of the major papilla [24].

\section{Statistical analysis}

A total of 96 subjects were projected to be required in the study to achieve a $95 \%$ confidence level and a margin of error of $7 \%$ to determine an $87 \%$ overall clinical success rate of EUS-BD [25]. Results are reported as mean \pm standard deviation (SD)/range for quantitative variables, and as percentages (\%) for categorical variables. Outcomes of pre- and post-EUS-BD bilirubin levels were compared using the paired Student's $t$ test/Wilcoxon signedrank test. Comparison of linear variables was done using Student's $t$ test/Mann-Whitney and categorical variables using Chi-square/Fisher's exact test. For clinical success, per protocol analysis was performed taking into account technically successful procedures and follow-up for at least 4 weeks, and intention to treat taking into account all cases. Kaplan-Meier analysis was performed to estimate patient survival and stent patency. Comparisons of stent patency were analyzed by the log rank test. 

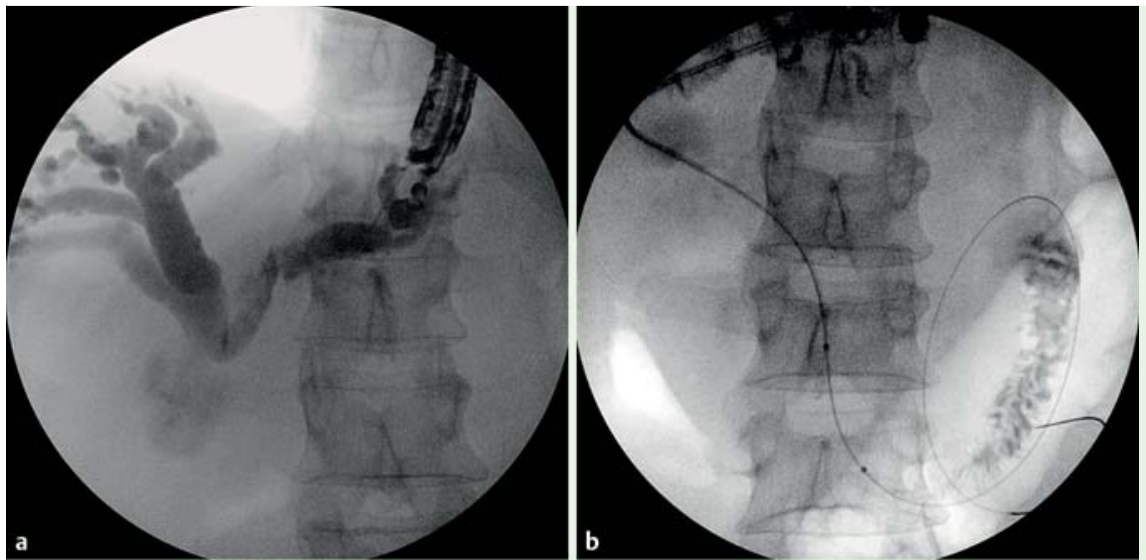

Fig.4 Endoscopic ultrasound-guided biliary drainage (EUS-BD) using the AGS approach in a patient with a large mass in the head of the pancreas causing biliary obstruction. a Fluoroscopic image of puncture of the left main hepatic duct and cholangiogram demonstrating an absence of filling in the extrahepatic bile duct. b Guidewire passed through the stricture into the duodenum (confirmed by enterogram). $\mathbf{c}$ Dilation of biliary stricture to facilitate self-expandable metallic stent (SEMS) placement. d Fluoroscopic image of SEMS deployed across the stricture.
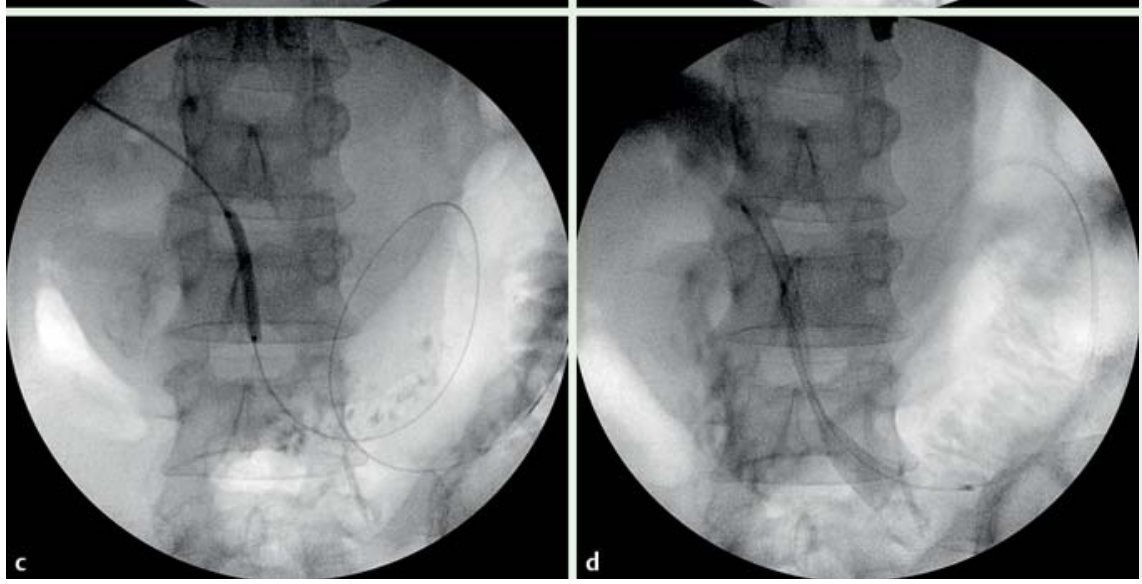

Overall survival was calculated from the date of EUS-BD procedure to the date of death, and living patients were censored at the date of last follow-up.Stent patency was calculated from the time of stent insertion to stent occlusion or migration. Patients who had not experienced stent occlusion or migration were censored at the date of last follow-up or date of death. Fisher's exact test was used to compare the proportions of stent occlusion/migration between EUS-BD using the transluminal approach and other techniques; and between HGS and other techniques. Statistical significance was based on two-sided design-based tests evaluated at $\alpha=0.05$. Statistical analysis was performed using SPSS version 21 (SPSS Inc, Chicago, IL, USA).

\section{Results}

During the study period, a total of 96 patients were enrolled at 12 tertiary centers ( Table $\mathbf{1})$. The mean age of participants was 66 years (range 33-95 years) and the majority were men $(n=53,55$ $\%)$. The most common etiology for malignant obstruction was pancreatic cancer, which accounted for $55 \%(n=53)$ of cases. Other etiologies included metastatic carcinoma $(n=15)$, cholangiocarcinoma $(n=9)$, ampullary cancer $(n=9)$, gastric cancer $(n=$ $2)$, lymphoma $(n=2)$, duodenal cancer $(n=1)$, and others $(n=4)$. Gastric outlet obstruction was present in 27 (28.1\%) patients: type I duodenal obstruction in 2, type II in 23, and type III in 2 patients. Post-surgical altered upper gastrointestinal anatomy was present in $10(10.4 \%)$ patients and included Billroth II $(\mathrm{n}=$ $3)$, Roux-en-Y hepaticojejunostomy $(n=2)$, pancreaticoduodenectomy $(n=1)$, and others $(n=4)$. Specific reasons for prior failed ERCP procedures were as follows: ampulla obscured by invasive cancer ( $n=43$ ), ampulla obscured by duodenal stents $(n=7)$, surgical upper gastrointestinal anatomy $(n=10)$, failed deep biliary cannulation $(n=20)$, and gastric outlet obstruction without in situ duodenal stents $(n=16)$. The mean preprocedural total bilirubin was $14 \mathrm{mg} / \mathrm{dL}( \pm 8.87)$.

EUS-BD was attempted in all 96 patients. Mean bile duct diameter that was punctured was $15.6 \mathrm{~mm}$ (range $3-30 \mathrm{~mm}$ ). EUSguided cholangiography was successful in all 96 (100\%) patients. EUS-guided fistula creation/dilation was performed using electrocautery in 43 (46.7\%) patients. Mean diameter of maximum tract dilation before stent placement was 7.6 Fr (range 4-22 Fr). Balloon tract dilation was used in 14 (14.5\%) patients. Successful stent placement (technical success) was achieved in a total of 92 (95.8\%) patients. The majority of patients underwent placement of biliary SEMS ( $n=84$ ), while a total of eight patients underwent placement of plastic biliary stents. Mean diameter of SEMS was $9.08 \mathrm{~mm}( \pm 1.84 \mathrm{~mm})$ and included 44 fully covered, 26 partially covered and 14 uncovered SEMS. The mean diameter of plastic stents was $3.05 \mathrm{~mm}( \pm 0.5 \mathrm{~mm})$. Procedures during which a plastic stent was placed included 5 CDS, 2 rendezvous, and 1 HGS. A total of 36 patents underwent EUS-BD following an intrahepatic approach, while 56 patients underwent an extrahepatic approach. Specific EUS-BD techniques used in the 92 patients who underwent successful stent placement included 69 transluminal, 12 antegrade stenting (AGS), and 11 rendezvous procedures. Among the 69 patients who underwent transluminal EUS$\mathrm{BD}, 50$ had CDS, 15 had HGS, and 4 had HDS. The mean procedure time was $40 \mathrm{~min}$ (range $8-207 \mathrm{~min}$ ). Mean procedure time for patients who underwent rendezvous was $45 \mathrm{~min}$, AGS was $46.1 \mathrm{~min}, \mathrm{CDS} 31 \mathrm{~min}, \mathrm{HGS} 69 \mathrm{~min}$, and HDS $22 \mathrm{~min}$. Mean procedure time for patients who underwent transluminal EUS-BD 
Table 1 Patient and procedural characteristics.

\begin{tabular}{|c|c|}
\hline & $\begin{array}{l}\text { EUS-guided biliary drainage } \\
(n=96)\end{array}$ \\
\hline Age, mean (SD), years & $66(15)$ \\
\hline Female, $n(\%)$ & $43(44.7)$ \\
\hline Pancreatic cancer, n (\%) & $53(55.0)$ \\
\hline Bilirubin, mean (SD), mg/dL & $14(8.8)$ \\
\hline \multicolumn{2}{|l|}{ Reason for EUS-BD, n (\%) } \\
\hline Ampulla obscured by invasive cancer & $43(44.7)$ \\
\hline Ampulla obstructed by enteral stent & $7(7.4)$ \\
\hline Altered anatomy & $10(10.4)$ \\
\hline Failed deep biliary cannulation & $20(20.8)$ \\
\hline Gastric outlet obstruction & $16(16.7)$ \\
\hline Cholangiography success, n (\%) & $96(100.0)$ \\
\hline Technical success, $\mathrm{n}(\%)$ & $92(95.8)$ \\
\hline \multicolumn{2}{|l|}{ Technique, n (\%) } \\
\hline Antegrade & $12(13.0)$ \\
\hline Rendezvous & $11(12.0)$ \\
\hline CDS & $50(54.4)$ \\
\hline HGS & $15(16.3)$ \\
\hline HDS & $4(4.3)$ \\
\hline \multicolumn{2}{|l|}{ Approach, n (\%) } \\
\hline Intrahepatic & $36(39.1)$ \\
\hline Extrahepatic & $56(60.9)$ \\
\hline Electrocautery, n (\%) & $43(44.8)$ \\
\hline Coaxial & $25(58.1)$ \\
\hline Non-coaxial & $18(41.9)$ \\
\hline \multicolumn{2}{|l|}{ Stent placed, $\mathrm{n}(\%)$} \\
\hline Metal & $84(91.3)$ \\
\hline Plastic & $8(8.7)$ \\
\hline Clinical success, $\mathrm{n}$ & 86 \\
\hline Intention-to-treat, \% & 89.5 \\
\hline Per-protocol, \% & 96.6 \\
\hline Procedure time, mean (range), min & $40(8-207)$ \\
\hline Adverse events, $\mathrm{n}(\%)$ & $10(10.5)$ \\
\hline Mild & $4(4.2)$ \\
\hline Moderate & $4(4.2)$ \\
\hline Severe & $1(1.0)$ \\
\hline Fatal & $1(1.0)$ \\
\hline Stent occlusion/migration, n (\%) & $5(5.4)$ \\
\hline
\end{tabular}

EUS-BD, endoscopic ultrasound-guided biliary drainage; CDS, choledochoduodenostomy; HGS, hepatogastrostomy; HDS, hepatoduodenostomy.

(CDS, HGS or HDS) was $38.5 \mathrm{~min}$. Mean procedure time for patients who underwent CDS or HDS was significantly shorter than for patients who underwent HGS $(P<0.05$ for both comparisons). Mean procedure time was not different in patients who underwent cautery-assisted tract dilation and those who did not ( $40.4 \mathrm{~min}$ vs $40.06 \mathrm{~min}, P=0.96$ ).

A total of two patients were lost to follow-up and one patient died the following day due to procedural complications. Among the remaining 89 patients who achieved technical success, clinical success was attained in 86 patients ( $89.5 \%$, intention-to-treat; $96.6 \%$, per-protocol analysis). There was a significant decrease in bilirubin $(\mathrm{mg} / \mathrm{dL})$ at 4 weeks $(14 \pm 8.8$ vs. $1.86 \pm 1.4, P<0.0001)$ ( $\checkmark$ Fig. 5). A total of 10 (10.5\%) procedure-related adverse events occurred: pneumoperitoneum $(n=2)$, bile leak $(n=3)$, cholangitis $(n=2)$, retained sheared wire $(n=1)$, bleeding $(n=1)$, and perforation $(\mathrm{n}=1)$ : 4 graded as mild, 4 moderate, 1 severe, and 1 fatal (due to perforation) ( Table 2 ). One severe adverse event occurred where a bile leak ensued after a failed attempt at choledochoduodenostomy. Furthermore, one fatal complication due to perforation occurred during a rendezvous procedure. There were no predictive factors of clinical success or occurrence of adverse events (all $P>0.05$ ).

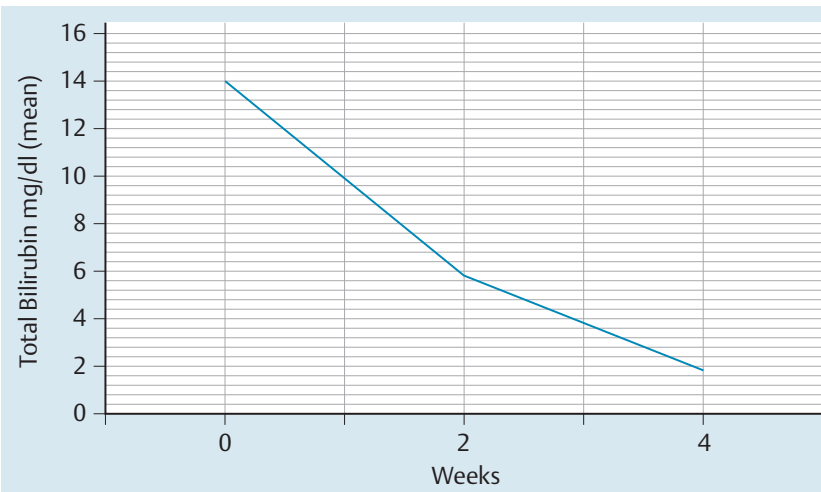

Fig. 5 Trend of decreasing bilirubin at 2 weeks and 4 weeks ( 2 weeks: $14 \pm 8.8$ vs. $5.8 \pm 9.7 \mathrm{mg} / \mathrm{dL}, P<0.0001 ; 4$ weeks: $14 \pm 8.8$ vs. $1.86 \pm 1.4 \mathrm{mg} /$ $\mathrm{dL}, P<0.0001)$.

- Table 3 details the characteristics and outcomes of patients who underwent EUS-BD using a transluminal approach (CDS, HGS or HDS) compared to those who underwent EUS-BD using rendezvous or AGS techniques. Baseline characteristics of patients in both groups were equivalent. Clinical success and adverse event rate were similar between both groups. Both procedure time and length of hospital stay were shorter in the transluminal group, although neither was statistically significantly different. During follow-up (median follow-up of the entire cohort was 94 days (range 9-741 days), there were significantly fewer stent occlusion events in the transluminal group (1.4\% vs. $13.6 \%$, $P=0.04$ ). The 1 -year stent patency was $87.5 \%$ (SE 0.11) in the rendezvous/AGS group vs $94.31 \%$ (SE 0.04) in the transluminal group (Log-rank test $P=0.73$ ) ( $\bullet$ Fig. 6).

- Table 4 details the characteristics and outcomes of patients who underwent EUS-BD using an intrahepatic approach compared to an extrahepatic approach. There was no difference is overall success (95\%CI 0.06-8.76) and adverse event rate between both approaches. However, an extrahepatic approach was significantly associated with decreased procedure time, length of hospital stay, and risk of moderate adverse events.

During the study period, recurrent biliary obstruction was observed in $5(5.4 \%)$ patients. This was the result of 4 stent occlusions and 1 stent migration requiring 5 re-intervention procedures: 1 new SEMS insertion, 1 balloon dilation, 1 ERCP, 1 PTBD, and 1 surgery. The 6-month stent patency rate was 95\% (95\% CI $94.94-95.06 \%)$ and the 1-year stent patency was $86 \%(95 \% \mathrm{CI}$ $85.74-86.26 \%$ ) ( Fig. 7). A total of 38 (44\%) out of 86 patients who had up-to-date available long-term follow-up data died of disease progression during the study period. The median overall patient survival was 167 days (95\%CI 131 - 372 days) after the index EUS-BD procedures ( $\bullet$ Fig.8).

\section{Discussion}

This is the first prospective, multicenter trial on EUS-BD and demonstrates the efficacy and safety of this technique in a large cohort of patients. A total of 96 patients underwent EUS-BD at 12 tertiary centers where procedures were performed by expert endoscopists. Technical success with appropriate stent placement was achieved in 92 (95.8\%) patients, 86 (96.6\%) of whom achieved clinical success with resolution of jaundice. A total of $10(10.5 \%)$ adverse events occurred, most (80\%) of which were 

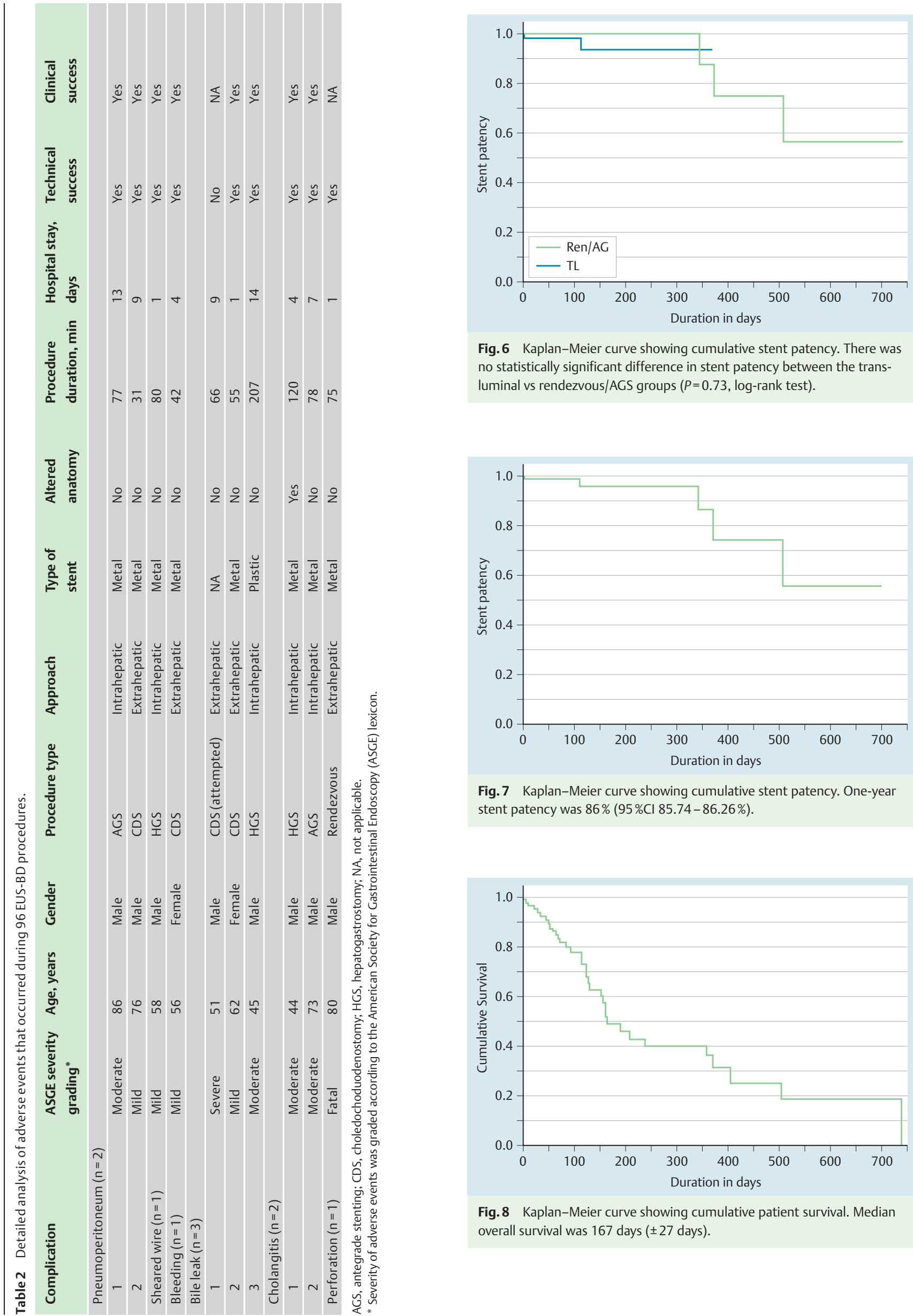

Fig. 6 Kaplan-Meier curve showing cumulative stent patency. There was no statistically significant difference in stent patency between the transluminal vs rendezvous/AGS groups ( $P=0.73$, log-rank test).

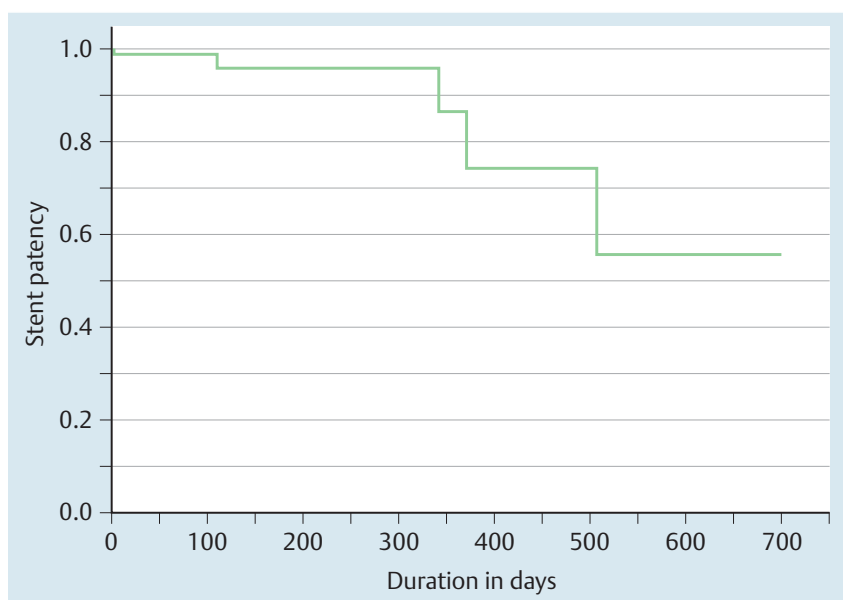

Fig.7 Kaplan-Meier curve showing cumulative stent patency. One-year stent patency was $86 \%$ ( $95 \% \mathrm{Cl} 85.74-86.26 \%)$.

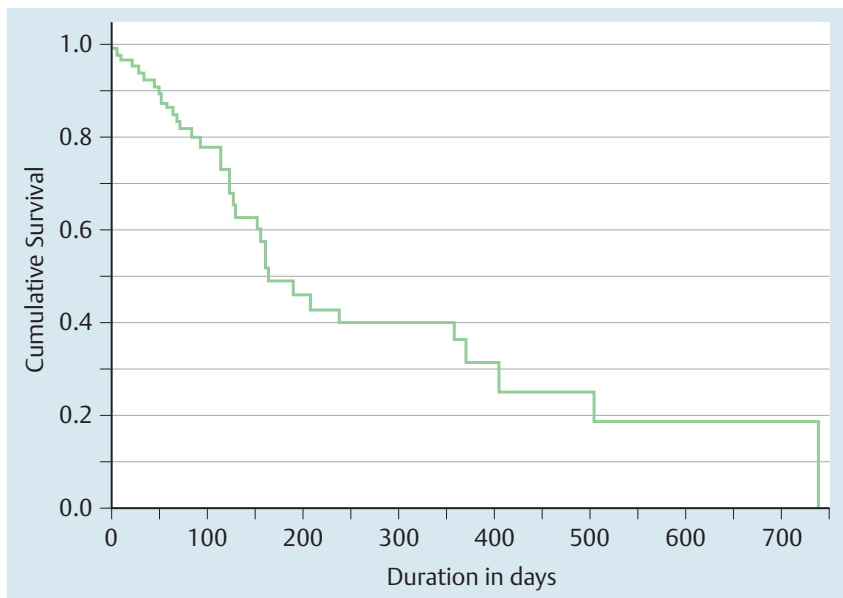

Fig. 8 Kaplan-Meier curve showing cumulative patient survival. Median overall survival was 167 days ( \pm 27 days). 


\begin{tabular}{|c|c|c|c|}
\hline & $\begin{array}{l}\text { Transluminal } \\
(n=69)\end{array}$ & $\begin{array}{l}\text { Rendezvous/\&AGS } \\
(n=23)\end{array}$ & $P$ value \\
\hline Age, mean (SD), years & $66.2(14.4)$ & $65.9(17.2)$ & 0.92 \\
\hline Female, $\mathrm{n}(\%)$ & $30(43.4)$ & $11(48.0)$ & 0.62 \\
\hline Maximal (SD) bile duct diameter, mm & $7.3(2.7)$ & $19(8.3)$ & 0.26 \\
\hline Mean (SD), pre-EUS-BD bilirubin, mg/dL & $13.8(8.1)$ & $14.3(11.1)$ & 0.82 \\
\hline Mean (SD), post-EUS-BD bilirubin 4 weeks, mg/dL & $1.8(1.4)$ & $1.8(1.8)$ & 0.97 \\
\hline Reduction in bilirubin, \% & $87 \%$ & $87.4 \%$ & 1.0 \\
\hline Electrocautery, $\mathrm{n}(\%)$ & $32(46.3)$ & $11(47.8)$ & 0.99 \\
\hline Clinical success, $\mathrm{n}(\%)$ & $64(92.7)$ & $22(95.6)$ & 0.99 \\
\hline Procedure time, mean (SD), min & $38.5(30.3)$ & $47.8(17.1)$ & 0.18 \\
\hline Length of hospital stay, mean (SD), days & $4(5.5)$ & $6.6(5.7)$ & 0.06 \\
\hline Adverse event, $\mathrm{n}(\%)$ & $6(8.6)$ & $3(13.0)$ & 0.68 \\
\hline Mild & $4(5.7)$ & $0(0.0)$ & 0.56 \\
\hline Moderate & $2(2.8)$ & $2(9.0)$ & 0.24 \\
\hline Severe/fatal & $0(0.0)$ & $1(4.3)$ & 0.24 \\
\hline Stent occlusion during long-term follow-up, $\mathrm{n}(\%)$ & $1(1.4)$ & $3(13.6)$ & $0.04^{*}$ \\
\hline Stent migration during long-term follow-up, $\mathrm{n}(\%)$ & $1(1.4)$ & $0(0.0)$ & 0.99 \\
\hline
\end{tabular}

Table 3 Comparison of baseline characteristics and outcomes of transluminal and rendezvous/ \&AGS techniques.

* $P<0.05$. AGS, antegrade stenting.

\begin{tabular}{|c|c|c|c|}
\hline & $\begin{array}{l}\text { Intrahepatic } \\
(n=36)\end{array}$ & $\begin{array}{l}\text { Extrahepatic } \\
(n=56)\end{array}$ & $P$ value \\
\hline Age, mean (SD), years & $61.8(16.2)$ & $68.9(13.6)$ & $0.03^{1}$ \\
\hline Female, $n(\%)$ & $16(44.4)$ & $25(44.6)$ & 0.99 \\
\hline Maximal (SD) bile duct diameter, mm & $7.8(3.7)$ & $7.3(3.1)$ & 0.61 \\
\hline Mean (SD), pre-EUS-BD bilirubin, mg/dL & $13.1(7.5)$ & $14.5(9.6)$ & 0.48 \\
\hline Mean (SD), post-EUS-BD bilirubin 4 weeks, mg/dL & $1.4(0.9)$ & $2.1(1.8)$ & 0.07 \\
\hline Reduction in bilirubin, \% & 89.3 & 85.5 & 1.0 \\
\hline Electrocautery, $\mathrm{n}(\%)$ & $19(52.7)$ & $24(42.8)$ & 0.40 \\
\hline Clinical success, $n(\%)$ & $34(94.4 \%)$ & $54(96.4)$ & 0.642 \\
\hline Procedure time, mean (SD), min & $54.3(34.9)$ & $31.8(18.1)$ & $0.001^{*}$ \\
\hline Length of hospital stay, mean (SD), days & $6.6(6.3)$ & $3.2(4.5)$ & $0.01^{1}$ \\
\hline Adverse event, $\mathrm{n}(\%)$ & $5(13.8)$ & $4(7.1)$ & 0.30 \\
\hline Mild & 1 & 3 & 1.0 \\
\hline Moderate & 4 & 0 & $0.02^{*}$ \\
\hline Severe/fatal & 0 & 1 & 1.0 \\
\hline Moderate/severe/fatal & 4 & 1 & 0.074 \\
\hline Stent occlusion during long-term follow-up, $\mathrm{n}(\%)$ & $3(8.3)$ & $1(1.7)$ & 0.29 \\
\hline Stent migration during long-term follow-up, $\mathrm{n}(\%)$ & $0(0.0)$ & $1(1.7)$ & 1.0 \\
\hline
\end{tabular}

Table 4 Comparison of baseline characteristics and outcomes of intrahepatic and extrahepatic groups. graded as either mild or moderate. Long-term outcomes were also favorable with a low rate of recurrent biliary obstruction during follow-up.

Despite growing international experience with EUS-BD in recent years, concern still remains about the safety and efficacy of these techniques compared to the standard, widely available alternative radiologic procedures. One source of concern is the scarcity of large prospective trials on EUS-BD. Data involving largely small series from expert centers suggest that EUS-BD can be performed with high therapeutic success (87\%) but is associated with 10 $20 \%$ morbidity (most mild - moderate) and rare serious adverse events [25]. The results of this international prospective trial are in accordance with other single center small prospective trials on EUS-BD. Artifon et al. published the first prospective, randomized trial comparing EUS-BD to PTBD in 25 patients, 13 of whom underwent EUS-CDS [26]. All procedures were technically and clinically successful (100\%). Adverse events occurred in $15.3 \%$ of patients. This small randomized study showed that EUS performed via a transluminal approach (choledochoduodenostomy) had a similar success rate, complication rate, and cost compared to
PTBD. Park and colleagues reported two prospective studies on EUS-BD. In their first study, they presented a relatively high adverse event rate of $20 \%$ [10] and in the more recent study [27] they aimed to evaluate whether a modified technique of "enhanced guidewire manipulation" could improve the safety and efficacy of EUS-BD. In this study, 45 patients with benign or malignant biliary obstruction underwent same session EUS-BD after failed ERCP. Technical success was achieved in 41 (91\%) patients and clinical success in 39 (95\%) of these patients. A total of 5 (11\%) adverse events occurred. Artifon and colleagues prospectively compared outcomes of EUS-CDS and EUS-HGS in 49 patients with malignant biliary obstruction and failed ERCP [28]. Overall, technical success, clinical success, and adverse events occurred in $94 \%, 85 \%$, and $16 \%$, respectively. Hamada et al. prospectively studied outcomes of EUS-BD in 18 patients and reported a technical success rate of $94 \%$, clinical success $100 \%$, and adverse event rate of $17 \%$ [29]. Our study, in line with recent published data, suggests that complication risk is decreasing as the experience with EUS-BD is growing. 
The current study also suggests excellent patency rates of stents placed during EUS-BD. The majority (91\%) of patients underwent placement of SEMS. Recurrent biliary obstruction was witnessed in only $5(5.4 \%)$ patients. The 6 -month and 12 -month stent patency rates were $95 \%$ and $86 \%$, respectively. Subgroup analysis also showed that there were significantly fewer stent occlusion events in patients who underwent EUS-BD using the transluminal approach compared to transpapillary (rendezvous/AGS) approaches $(P=0.04)$. The majority (75\%) of patients in this study underwent EUS-BD using a transluminal approach. This may at least partially explain the long-term stent patency rate in the study. Randomized trials assessing SEMS patency in biliary obstruction when all stents were placed via ERCP demonstrated an expected recurrent stent obstruction of about $50 \%$ at 1 year after stent placement [30]. Overall, the current study demonstrates an excellent long-term patency of stents placed during EUS-BD, specifically when a transluminal approach is undertaken. Hamada and colleagues compared stent patency in patients with indwelling duodenal stents who underwent stent placement via ERCP or EUS-BD using a transluminal approach. The stent patency rate in the EUS-BD group was higher than that in the transpapillary drainage group (100 vs. $71 \%$ at 1 month and 83 vs. $29 \%$ at 3 months, respectively). The rate of stent dysfunction in the EUSBD group tended to be lower than that in the transpapillary group (14 vs. $54 \%, P=0.16$ ). There are multiple hypothetical reasons for possibly improved stent patency outcomes during transluminal drainage compared to transpapillary drainage. Duodenobiliary reflux is a major contributor of recurrent obstruction of biliary stents and may occur less when stents are placed away from the ampulla (CDS and HGS) [29]. Furthermore, EUS-BD via a transluminal approach allows antegrade biliary drainage and bypass of the biliary obstruction site. This avoids turbulent biliary flow, stent compression by the tumor, and risk of tumor ingrowth/overgrowth.

EUS-BD performed using the rendezvous or AGS techniques is preferred over the transluminal techniques by many endoscopists as they avoid the need for a permanent biliary-enteric fistula and the need to dilate the fistulous tract, which may lead to complications such as bile leak, bleeding, pneumoperitoneum, and pneumomediastinum. However, EUS-BD using these techniques is frequently not possible as the wire cannot pass through the ampulla due to difficult angulation or tight distal biliary stricture [16]. Nonetheless, prior small retrospective studies have suggested EUS-BD using transluminal technique as an effective and safe alternative to the other techniques [31]. In the current study, transluminal EUS-BD was as effective and safe as rendezvous/AGS procedures. It is noteworthy that the majority of patients received SEMS, which seals the biliary-enteric fistula and, thus, protects against complications, especially bile leakage. We would recommend SEMS in favor of plastic stents where feasible. The optimal approach (intrahepatic vs extrahepatic) to EUS-BD is debatable and this comparison has not been studied prospectively. Retrospective studies have suggested that both approaches were equally effective but an extrahepatic approach was associated with a significantly decreased risk of adverse events [32]. In the current study, an extrahepatic approach was significantly associated with decreased procedure time, length of hospital stay, and risk of moderate adverse events. Overall, an extrahepatic approach resulted in $52 \%$ decreased risk of adverse events, although this result was not statistically significant (OR $0.48, P=$ 0.31 ) likely because the study was not powered to show this. There are multiple conjectural reasons that render the extrahe- patic approach to EUS-BD safer than the intrahepatic approach. An intrahepatic route involves needle puncture into the peritoneal cavity, which risks pneumoperitoneum and bile leakage. Also, the movement of the liver during respiration may lead to both stent migration with resulting bilomas and increased trauma to the bilioenteric tract. Finally, smaller caliber intrahepatic ducts may not allow placement of wider metallic stents, which can theoretically predispose to pneumoperitoneum and bile leakage due to incomplete sealing of the bilioenteric fistula.

This study has some limitations. All EUS-BD procedures were performed by experts and each had performed at least 20 prior successful EUS-BD procedures. Lower technical success rates and higher complication rates have been reported during the first 20 EUS-BD procedures [33]. Therefore, results from this study may not be generalizable. The study was also not powered to detect outcome differences between various EUS-BD techniques (e.g. transluminal vs. rendezvous/AGS) and approaches (intrahepatic and extrahepatic). Therefore, negative comparisons may represent type II errors.

In conclusion, this prospective international multicenter study on EUS-BD demonstrates the excellent efficacy and safety of EUS-BD when performed by experts.

Competing interests: Mouen Khashab is a consultant for Boston Scientific, Olympus America, and Xlumena. Schalk van der Merwe is a consultant for Boston Scientific and Cook Endoscopy. Manuel Perez-Miranda is a consultant for Boston Scientific and Xlumena.

\section{Institutions}

${ }^{1}$ Johns Hopkins Medical Institute, Baltimore, MD, USA

2 Department of Hepatology, Division of Liver, and Pancreatico-Biliary Disorders, University Hospital Gastuisberg, University of Leuven, Leuven Belgium ${ }^{3}$ Aarhus University Hospital, Aarhus, Denmark

${ }^{4}$ Department of Surgery, The Chinese University of Hong Kong, Prince of Wales Hospital, Hong Kong SAR, China

${ }^{5}$ Department of Surgery, University of Sao Paulo, Sao Paulo, Brazil

${ }^{6}$ Unit of Gastroenterology and Digestive Endoscopy, AUSL Bologna

Bellaria-Maggiore Hospital, Bologna, Italy

${ }^{7}$ Endoscopy Service, Department of Diagnostic and Therapeutic Services,

Mediterranean Institute for Transplantation and Advanced Specialized

Therapies (ISMETT), Palermo, Italy

${ }^{8}$ Center for Interventional Endoscopy, Florida Hospital, Orlando, FL, USA

${ }^{9}$ Winthrop University Hospital, Mineola, NY, USA

${ }^{10}$ Hospital Universitario Rio Hortega, Valladolid, Spain

${ }^{11}$ Division of Gastroenterology and Hepatology, Medical University of South Carolina, Charleston, SC, USA

${ }^{12}$ Division of Gastroenterology and Hepatology, University of Colorado Anschutz Medical Campus, Aurora, CO, USA

\section{References}

1 Khashab MA, Varadarajulu S. Endoscopic ultrasonography as a therapeutic modality. Curr Opin Gastroenterol 2012; 28: 467-476

2 Khashab MA, Valeshabad AK, Afghani E et al. A comparative evaluation of EUS-guided biliary drainage and percutaneous drainage in patients with distal malignant biliary obstruction and failed ERCP. Dig Dis Sci 2015; 60: 557-565

3 Wiersema M. Endosonography-guided cholangiopancreatography. Gastrointest Endosc 1996; 44: $102-106$

4 Giovannini M, Moutardier V, Pesenti $C$ et al. Endoscopic ultrasoundguided bilioduodenal anastomosis: a new technique for biliary drainage. Endoscopy 2001; 33: 898-900

5 Kahaleh M, Hernandez AJ, Tokar J et al. Interventional EUS-guided cholangiography: evaluation of a technique in evolution. Gastrointest Endosc 2006; 64: 52-59

6 Maranki J, Hernandez AJ, Arslan B et al. Interventional endoscopic ultrasound-guided cholangiography: long-term experience of an emerging alternative to percutaneous transhepatic cholangiography. Endoscopy 2009; 41: $532-538$ 
7 Kim YS, Gupta K, Mallery S et al. Endoscopic ultrasound rendezvous for bile duct access using a transduodenal approach: cumulative experience at a single center. A case series. Endoscopy 2010; 42: 496-502

8 Fabbri C, Luigiano C, Fuccio L et al. EUS-guided biliary drainage with placement of a new partially covered biliary stent for palliation of malignant biliary obstruction: a case series. Endoscopy 2011; 43: 438441

9 Komaki T, Kitano M, Sakamoto $H$ et al. Endoscopic ultrasonographyguided biliary drainage: evaluation of a choledochoduodenostomy technique. Pancreatology 2011; 11: 47-51

10 Park DH, Jang JW, Lee SS et al. EUS-guided biliary drainage with transluminal stenting after failed ERCP: predictors of adverse events and long-term results. Gastrointest Endosc 2011; 74: 1276 - 1284

11 Hara K, Yamao K, Niwa Y et al. Prospective clinical study of EUS-guided choledochoduodenostomy for malignant lower biliary tract obstruction. Am J Gastroenterol 2011; 106: 1239-1245

12 Iwashita T, Lee JG, Shinoura S et al. Endoscopic ultrasound-guided rendezvous for biliary access after failed cannulation. Endoscopy 2012; 44: $60-65$

13 Khashab M, Fujii LL, Baron TH et al. EUS-guided biliary drainage for patients with malignant biliary obstruction with an indwelling duodenal stent (with video). Gastrointest Endosc 2012; 76: 209-213

14 Henry WA, Singh VK, Kalloo AN et al. Simultaneous EUS-guided transbulbar pancreaticobiliary drainage (with video). Gastrointest Endosc 2012; 76: 1065-1067

15 Dhir V, Bhandari S, Bapat $M$ et al. Comparison of EUS-guided rendezvous and precut papillotomy techniques for biliary access (with videos). Gastrointest Endosc 2012; 75: 354-359

16 Shah JN, Marson F, Weilert F et al. Single-operator, single-session EUSguided anterograde cholangiopancreatography in failed ERCP or inaccessible papilla. Gastrointest Endosc 2012; 75: 56-64

17 Itoi T, Yamao K. EUS 2008 Working Group document: evaluation of EUS-guided choledochoduodenostomy (with video). Gastrointest Endosc 2009; 69: 8-12

18 Savides TJ, Varadarajulu S, Palazzo L. EUS 2008 Working Group document: evaluation of EUS-guided hepaticogastrostomy. Gastrointest Endosc 2009; 69: 3-7

19 Artifon EL, Safatle-Ribeiro AV, Ferreira FC et al. EUS-guided antegrade transhepatic placement of a self-expandable metal stent in hepaticojejunal anastomosis. JOP 2011; 12: 610-613

20 Nguyen-Tang T, Binmoeller KF, Sanchez-Yague A et al. Endoscopic ultrasound (EUS)-guided transhepatic anterograde self-expandable metal stent (SEMS) placement across malignant biliary obstruction. Endoscopy 2010; 42: 232-236
21 Saxena P, Kumbhari V, Zein ME et al. EUS-guided biliary drainage with antegrade transpapillary placement of a metal biliary stent. Gastrointest Endosc 2015; 81: 1010 - 1011

22 Kumbhari V, Tieu AH, Khashab MA. EUS-guided biliary drainage made safer by a combination of hepaticogastrostomy and antegrade transpapillary stenting. Gastrointest Endosc 2015; 81: 1015-1016

23 Cotton PB, Eisen GM, Aabakken $L$ et al. A lexicon for endoscopic adverse events: report of an ASGE workshop. Gastrointest Endosc 2010; 71: $446-454$

24 Mutignani M, Tringali A, Shah SG et al. Combined endoscopic stent insertion in malignant biliary and duodenal obstruction. Endoscopy 2007; 39: $440-447$

25 Khashab MA, Dewitt J. EUS-guided biliary drainage: is it ready for prime time? Yes! Gastrointest Endosc 2013; 78: 102-105

26 Artifon EL, Aparicio D, Paione JB et al. Biliary drainage in patients with unresectable, malignant obstruction where ERCP fails: endoscopic ultrasonography-guided choledochoduodenostomy versus percutaneous drainage. J Clin Gastroenterol 2012; 46: 768 - 774

27 Park do H, Jeong $S U$, Lee $B U$ et al. Prospective evaluation of a treatment algorithm with enhanced guidewire manipulation protocol for EUSguided biliary drainage after failed ERCP (with video). Gastrointest Endosc 2013; 78: 91 - 101

28 Artifon EL, Marson FP, Gaidhane M et al. Hepaticogastrostomy or choledochoduodenostomy for distal malignant biliary obstruction after failed ERCP: Is there any difference? Gastrointest Endosc 2015; 81: 950-959

29 Hamada T, Isayama H, Nakai Y et al. Transmural biliary drainage can be an alternative to transpapillary drainage in patients with an indwelling duodenal stent. Dig Dis Sci 2014; 59: 1931 -1938

30 Telford JJ, Carr-Locke DL, Baron TH et al. A randomized trial comparing uncovered and partially covered self-expandable metal stents in the palliation of distal malignant biliary obstruction. Gastrointest Endosc 2010; 72: 907-914

31 Khashab MA, Valeshabad AK, Modayil R et al. EUS-guided biliary drainage by using a standardized approach for malignant biliary obstruction: rendezvous versus direct transluminal techniques (with videos). Gastrointest Endosc 2013; 78: 734-741

32 Dhir V, Artifon EL, Gupta K et al. Multicenter study on endoscopic ultrasound-guided expandable biliary metal stent placement: Choice of access route, direction of stent insertion, and drainage route. Dig Endosc 2014; 26: 430-435

33 Vila JJ, Perez-Miranda M, Vazquez-Sequeiros $E$ et al. Initial experience with EUS-guided cholangiopancreatography for biliary and pancreatic duct drainage: a Spanish national survey. Gastrointest Endosc 2012; 76: $1133-1141$ 